\title{
Pulmonary vascular effects of pulsed inhaled nitric oxide in COPD patients with pulmonary hypertension
}

\author{
Bita Hajian' \\ Jan De Backer ${ }^{2}$ \\ Wim Vos ${ }^{2}$ \\ Cedric Van Holsbeke ${ }^{2}$ \\ Francisca Ferreira ${ }^{2}$ \\ Deborah A Quinn ${ }^{3}$ \\ Annemie Hufkens' \\ Rita Claes' \\ Wilfried De Backer' \\ 'Department of Respiratory Medicine, \\ University Hospital Antwerp, Edegem, \\ ${ }^{2}$ FluidDA nv, Antwerp, Belgium; \\ ${ }^{3}$ Bellerophon Therapeutics, Warren, \\ NJ, USA
}

This article was published in the following Dove Press journal: International Journal of COPD

5 July 2016

Number of times this article has been viewed
Introduction: Severe chronic obstructive pulmonary disease (COPD) is often associated with secondary pulmonary hypertension $(\mathrm{PH})$, which worsens prognosis. $\mathrm{PH}$ can be lowered by oxygen, but also by inhaled nitric oxide (NO), which has the potential to improve the health status of these patients. NO is an important mediator in vascular reactions in the pulmonary circulation. Oral compounds can act through NO-mediated pathways, but delivering pulsed inhaled NO (iNO) directly to the airways and pulmonary vasculature could equally benefit patients. Therefore, a proof-of-concept study was performed to quantify pulmonary blood vessel caliber changes after iNO administration using computed tomography (CT)-based functional respiratory imaging (FRI).

Methods: Six patients with secondary PH due to COPD received "pulsed" iNO in combination with oxygen for 20 minutes via a nasal cannula. Patients underwent a high-resolution CT scan with contrast before and after iNO. Using FRI, changes in volumes of blood vessels and associated lobes were quantified. Oxygen saturation and blood pressure were monitored and patients were asked about their subjective feelings.

Results: Pulmonary blood vessel volume increased by $7.06 \% \pm 5.37 \%$ after iNO. A strong correlation $\left(\Omega^{2}=0.32, P=0.002\right)$ was obtained between ventilation and observed vasodilation, suggesting that using the pulsed system, iNO is directed toward the ventilated zones, which consequently experience more vasodilation. Patients did not develop oxygen desaturation, remained normotensive, and perceived an improvement in their dyspnea sensation.

Conclusion: Inhalation of pulsed NO with oxygen causes vasodilation in the pulmonary circulation of COPD patients, mainly in the well-ventilated areas. A high degree of heterogeneity was found in the level of vasodilation. Patients tend to feel better after the treatment. Chronic use trials are warranted.

Keywords: pulmonary hypertension, COPD, pulsed inhaled nitric oxide, FRI

\section{Introduction}

An estimated $30 \%-70 \%$ of patients with chronic obstructive pulmonary disease (COPD) have pulmonary hypertension (PH). ${ }^{1} \mathrm{PH}$ associated with COPD is classified as Group $3 \mathrm{PH}$ by the World Health Organization (WHO), which is associated with lung disease and/or hypoxemia. ${ }^{2}$ The relevance of a vascular pathology in COPD is still somewhat unresolved, ${ }^{3,4}$ but there is clear evidence that PH plays an important role in the morbidity and mortality associated with COPD. The prognosis of COPD with severe $\mathrm{PH}$ and a resting pulmonary artery pressure (PAP) $>35-40 \mathrm{mmHg}^{5,6}$ is very poor. Systemic treatment of PH causes a lot of side effects: these drugs cause systemic hypotension as they lower the pulmonary and systemic vascular resistance
Correspondence: Bita Hajian

Department of Respiratory Medicine,

University Hospital Antwerp,

Wilrijkstraat 10, Edegem 2650, Belgium

Tel +32 38213000

Email bita.hajian@uza.be 
and worsen the mismatch of ventilation perfusion in the lung. ${ }^{7}$ Survival improves in COPD patients with secondary $\mathrm{PH}$ by long-term oxygen therapy (LTOT). ${ }^{8}$ There is evidence that in hypoxic lung diseases, there is impaired endothelial cell release of nitric oxide (NO). ${ }^{9}$ Inhaled NO is currently under development for other potential clinical indications because it is a powerful endothelium-derived relaxing factor. ${ }^{10-13}$ Inhaled NO therefore seems to be both a selective and an effective pulmonary vasodilator. ${ }^{12}$ By administering NO through inhalation, it is directly delivered to the target organ, which allows for a lower dose than is necessary with systemic delivery, thereby resulting in fewer and less severe adverse effects. Pulsed inhalation of NO with oxygen is a safe and effective treatment for patients with COPD and $\mathrm{PH} .{ }^{14}$

Inhaled NO, a prescription pharmaceutical drug under the brand name iNOmax ${ }^{\circledR}$ (NO; INO Therapeutics, Madison, WI, USA) for inhalation, is available commercially as a gaseous mixture of NO and nitrogen $\left(\mathrm{N}_{2}\right)$. iNOmax is approved in the US (December 1999), European Union (August 2001), and other national entities for neonates ( $>34$ weeks old) with hypoxic respiratory failure associated with clinical or echocardiographic evidence of PH when used in conjunction with ventilatory support and other appropriate agents. ${ }^{15-18}$ To our knowledge, there are no studies looking at the vessel caliber of the lung during NO treatment. The aim of our study is to investigate the feasibility, safety, and acute therapeutic effect of pulsed NO using functional respiratory imaging to calculate the changes in blood vessel caliber in patients with COPD with $\mathrm{PH}$.

\section{Patients and methods}

\section{Patients}

Patients with a confirmed diagnosis of COPD (according to the Global initiative for chronic Obstructive Lung Disease [GOLD] criteria with a postbronchodilator forced expiratory volume in 1 second $\left(\mathrm{FEV}_{1}\right) /$ forced vital capacity $(\mathrm{FVC})<0.7$ and an $\mathrm{FEV}_{1}$ value $<60 \%$ predicted) and using LTOT for $>10$ hours a day, were included in this study. PH was determined within the previous 12 months by a right heart catheterization with a mean PAP $(\mathrm{mPAP}) \geq 25 \mathrm{mmHg}$, or an echocardiogram with a tricuspid regurgitant velocity $\geq 2.9 \mathrm{~m} / \mathrm{s}$ or systolic PAP (sPAP) $\geq 38 \mathrm{mmHg}$. Patients had to be between 40 years and 80 years of age. Patients were excluded from the study if they had any of the following: an exacerbation during the past month requiring the start of or increase in systemic oral corticosteroid therapy and/or hospitalization, left ventricular dysfunction with echocardiographic evidence of a left ventricular ejection fraction $(\mathrm{LVEF})<40 \%$. Also excluded were patients with moderate LV diastolic dysfunction (ie, $>$ Grade
2) or any history of increased pulmonary capillary wedge pressure, left atrial pressure, or left ventricular end diastolic pressure $>18 \mathrm{mmHg}$ as measured during cardiac catheterization within the past 6 months. In addition, patients with renal impairment (ie, an estimated glomerular filtration rate (GFR) Modification of Diet in Renal Disease (MDRD) study value $<60 \mathrm{~mL} / \mathrm{min} / 1.73 \mathrm{~m}^{2}$ ) or history of renal failure, those with clinically significant valvular heart disease that may contribute to $\mathrm{PH}$, and patients using an approved $\mathrm{PH}$ medication, such as sildenafil or bosentan, currently or within 30 days of screening were excluded.

\section{NO delivery system}

For the indication of COPD-associated $\mathrm{PH}$, inhaled nitrogen oxide (iNO) is being developed as a drug/device combination product to be used with the investigational INOpulse ${ }^{\circledR}$ DS-C delivery device (Bellerophon Therapeutics, Warren, NJ, USA). The investigational INOpulse ${ }^{\circledR}$ DS-C delivery system is made up of a device that uses $0.16 \mathrm{~L}$ mini cylinders containing $3.0 \mathrm{mg} / \mathrm{L}(2,440 \mathrm{ppm})$ or $6.0 \mathrm{mg} / \mathrm{L}(4,880 \mathrm{ppm})$ of NO gas (Figure 1). The device is lightweight, portable, and can be used in an ambulatory setting. The main advantage of the INOpulse DS-C device for spontaneously breathing patients lies in its ability to deliver precise, preset iNO doses over time, independent of the patients' respiration rate and tidal volume. Prescribed doses of iNO are delivered through INOpulse DS-C device according to the ideal body weight (IBW) per hour (micrograms/kilogram IBW/hour). The iNO dose is pulsed during the beginning of the subject's inspiration rather than throughout the entire inspiratory period, and the hourly set dose is accurately delivered each hour. Because the amount of drug delivered by the INOpulse DS-C device is independent of the subject's respiratory frequency and tidal volume, the dose of iNO is tightly controlled.

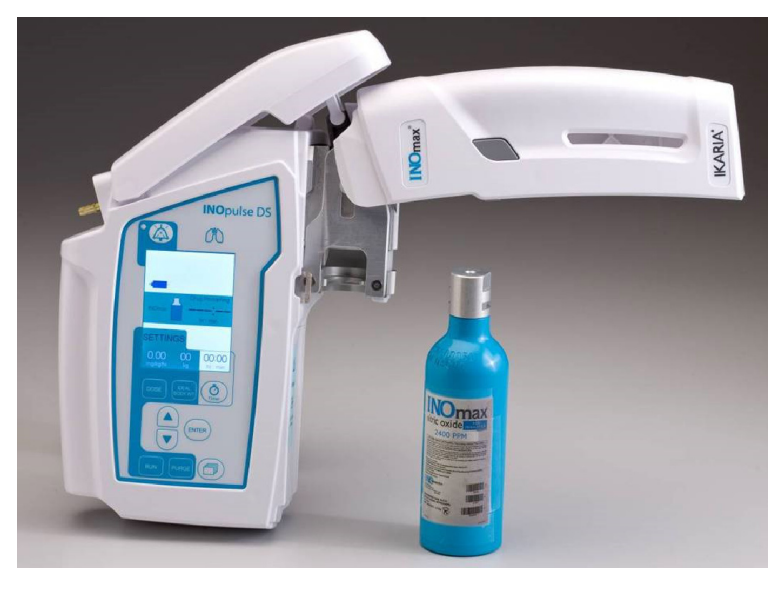

Figure I INOpulse DS-C delivery device and cartridge. Abbreviation: iNO, inhaled nitric oxide. 
A direct comparison between pulsatile delivery and constant concentration delivery is difficult as the delivered dose varies with the breath rate for constant concentration delivery, while it is constant for the pulsatile delivery of the INOpulse device. The level of exposure between pulsatile and constant concentration deliveries can be compared by making assumptions regarding respiration rate and tidal volume, as well as the IBW, for the INOpulse device.

Patients received $30 \mu \mathrm{g} / \mathrm{kg}$ IBW/hour doses (INOpulse DS-C setting of $0.030 \mathrm{mg} / \mathrm{kg}$ IBW/hour). One advantage of pulsed delivery of iNO early in inspiration is that it presumably delivers the drug selectively to the healthiest wellventilated lung segments by using a short pulse width.

\section{Study protocol and patient characteristics}

The objective of this exploratory study was to assess the effect of pulsed iNO on vascular geometry in subjects with COPD-related PH who are on LTOT. The primary end point was the change in lobar blood volume at total lung capacity (TLC), measured by functional respiratory imaging (FRI), after dosing with pulsed iNO. Additional end points were internal airflow distribution (to link regional vasodilation with regional ventilation) and patient feeling of dyspnea and exercise tolerance. The study protocol (Figure 2) was approved by the Ethical Committe of the University Hospital of Antwerp, (14/35/361) and informed consent was given by each patient at the time of entry to the study. The clinical trial registration number for this study is NCT02267655.

Six patients (three males and three females) with adequate renal function (estimated $\mathrm{GFR}_{\mathrm{MDRD}}$ ) $\geq 60 \mathrm{~mL} / \mathrm{min} / 1.73 \mathrm{~m}^{2}$ and meeting all inclusion and none of the exclusion criteria were enrolled (Table 1). At screening, treatment visit, and posttreatment, the following were measured: vital signs [heart rate (HR), respiratory rate (RR), noninvasive blood pressure (NIBP) and oxygen partial pressure $\left.\left(\mathrm{SpO}_{2}\right)\right]$ and methemoglobin (metHb) (via pulse oximeter). After NO treatment also, the spirometry was performed.

Four low-dose high-resolution computed tomography (HRCT) scans were obtained while the patient was on LTOT and room air for at least 20 minutes (baseline phase). Two baseline TLC scans were taken quickly after each other with contrast, given before the first TLC scan. These two baseline HRCT scans were performed at TLC to demonstrate baseline variability and/or repeatability. Next, one baseline functional residual capacity (FRC) scan and one upper airway CT scan was performed. After the patient received $30 \mu \mathrm{g} / \mathrm{kg}$ IBW/hour, the patient remained lying down, not removed from the motorized bed of the CT scanner, starting at least 20 minutes before the baseline CT scans were taken. $\mathrm{MetHb}$ measurement and vital signs were obtained before discontinuing dosing with iNO. The baseline TLC and FRC scans (preceding the iNO dose) were used to compute the baseline values for the primary end point (ie, blood vessel density) and the secondary end points (ie, internal airflow distribution).

\section{Functional respiratory imaging}

In the FRI workflow, CT images are converted into threedimensional images of the lung lobes, the airway tree, and vessels. ${ }^{19-21}$ By segmenting the lung lobes at FRC and TLC

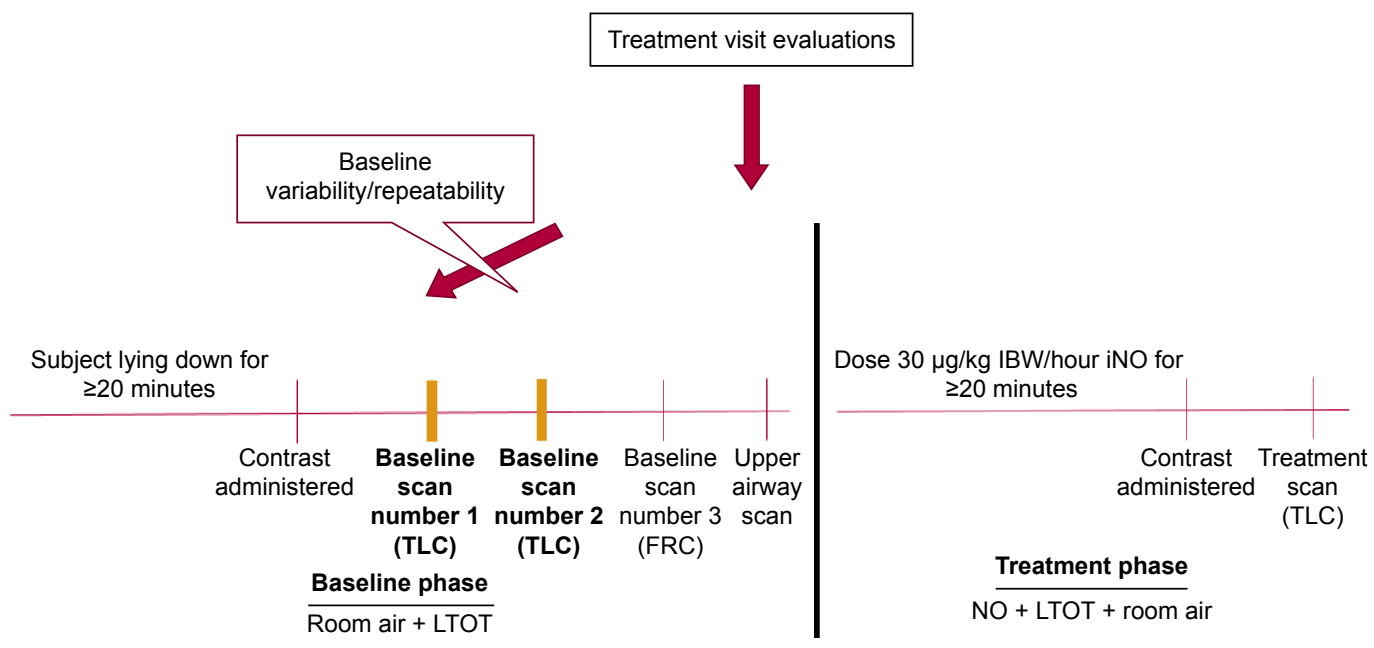

Figure 2 Study protocol.

Note: LTOT is represented in L/min.

Abbreviations: FRC, functional residual capacity; IBW, ideal body weight; iNO, inhaled nitric oxide; LTOT, long-term oxygen therapy; NO, nitric oxide; TLC, total lung capacity. 
Table I Patient characteristics

\begin{tabular}{|c|c|c|c|c|c|c|}
\hline Characteristics & Patient I & Patient 2 & Patient 3 & Patient 4 & Patient 5 & Patient 6 \\
\hline Age (years) & 67 & 68 & 76 & 76 & 68 & 79 \\
\hline Sex & $\mathrm{F}$ & $M$ & $\mathrm{~F}$ & M & $M$ & $\mathrm{~F}$ \\
\hline Height (cm) & 164 & 160 & 156 & 165 & 178 & 160 \\
\hline Weight (kg) & 55 & 89 & 51 & 60 & 47 & 50 \\
\hline $\mathrm{FEV}_{1}$ (\%pred) pre-NO & 0.74 & 0.87 & 0.84 & 0.59 & 0.77 & 0.61 \\
\hline $\mathrm{FEV}_{\text {, }}(\%$ pred) post-NO & 0.49 & 0.77 & 0.87 & 0.59 & 0.8 & 0.56 \\
\hline FVC (L) pre-No & 2.52 & 2.78 & 2.32 & 2.96 & 2.7 & 2.16 \\
\hline FVC (L) post-NO & 1.92 & 2.3 & 2.4 & 3.25 & 2.9 & 2.03 \\
\hline $\mathrm{FEV}_{\mathrm{I}} / \mathrm{FVC}(\%)$ pre-NO & 29 & 34 & 36 & 20 & 29 & 28 \\
\hline $\mathrm{FEV}_{\mathrm{l}} / \mathrm{FVC}(\%)$ post-NO & 25 & 33 & 36 & 18 & 28 & 28 \\
\hline LTOT (L/min) & 2 & 2 & 2 & 2 & 1.5 & 2 \\
\hline Hours LTOT/d & 24 & 24 & 16 & 24 & 22 & 16 \\
\hline Smoking history (pack-years) & 25.5 & 90 & 25.8 & 50 & 57.5 & 71.25 \\
\hline $\mathrm{SpO}_{2}(\%)$ pre-NO & 96 & 98 & 99 & 95 & 94 & 96 \\
\hline $\mathrm{SpO}_{2}(\%)$ post-NO & 97 & 98 & 99 & 96 & 94 & 100 \\
\hline sPAP $(\mathrm{mmHg})$ screening & 52 & 47 & 44 & 39 & 56 & 43 \\
\hline Blood pressure (mmHg) pre-NO & $135 / 80$ & $110 / 60$ & $116 / 62$ & $130 / 70$ & $122 / 70$ & $152 / 80$ \\
\hline Blood pressure $(\mathrm{mmHg})$ post-NO & $140 / 86$ & $134 / 74$ & $170 / 80$ & $170 / 80$ & $120 / 70$ & $158 / 80$ \\
\hline MethHb pre-NO & 0.6 & 0.4 & 0.8 & 0 & 0.4 & 0.9 \\
\hline MethHb post-NO & 0.6 & 0.3 & 0.2 & 0 & 0.5 & 0.7 \\
\hline
\end{tabular}

Abbreviations: \%pred, percentage of predicted; F, female; FEV , forced expiratory volume in I second; FEV,/FVC, Tiffeneau-Pinelli index; FVC, forced vital capacity; LTOT, long-term oxygen therapy; M, male; NO, nitric oxide; post-NO, post-nitric oxide inhalation; pre-NO, pre-nitric oxide inhalation; SpO ${ }_{2}$, oxygen partial pressure; sPAP, systolic pulmonary arterial pressure; methHb, methemoglobin.

(Video S1), the internal airflow distribution can be derived from the relative volume change between these two lung volumes. The airway tree is presented at the TLC level. At TLC, the airway structure can be segmented down to bronchi with diameter of about 1-2 $\mathrm{mm}$. Beyond this point, the CT resolution is insufficient to distinguish alveolar and intraluminal air. A typical airway model includes five to ten generations, depending mainly on the disease state of the individual patient. Distal airway volumes (iVaw) can be assessed at individual airways and in different regions. Anatomical structures that were analyzed include lung volumes, lobar volumes, and local airway volumes, mainly lung blood vessels volumes that were obtained before and after iNO administration. Based on this, we can describe regional flow distribution. The contrast CT scans make it possible to extract the blood vessels by performing Hounsfield thresholding, combined with region growing, starting from the central vessels. The HRCT parameters are extracted using a semiautomated tool (Mimics 15.0, Materialise NV, Leuven, Belgium; Food and Drug Administration, K073468; Conformité Européenne certificate, BE 05/1191. CE.01), which identified the fissures separating the lung lobes. For the calculation of vasodilation, only blood vessels that are present in both scans were considered.

\section{Statistical analysis}

All statistical analyses were conducted using $\mathrm{R}$ version 3.0.2 (The R Foundation for Statistical Computing, Vienna,
Austria). The significance level was set at 0.05 . The global differences before and after the treatment phase were assessed using a paired $t$-test. The data were also analyzed on a lobar level using linear mixed-effects models, whereby different subjects are combined in a random factor. In this mixedeffect approach, goodness of fit is described using $\Omega^{2}{ }_{0}$, as proposed by $\mathrm{Xu}^{22}$

\section{Results}

Changes in blood vessel caliber could be observed in all patients, as shown in Figure 3. The color code indicates the changes from baseline. The vasodilation is heterogeneous (Figure 4), but the overall effect is that there is big improvement in the volume of the vessels. In some areas, the blood vessel volume increased $>20 \%$ compared to the baseline value.

Figure 5 shows the vascular changes for the total lung and for the individual lobes. In addition, the figure depicts the variability of the FRI measurement $(-1.56 \% \pm 3.57 \%)$ relative to the treatment effect $(7.06 \% \pm 5.88 \%)$. It can be observed that the variability of the measurement, as determined using the test-retest scenario, is small compared to the iNO treatment effect. There is a significant vasodilation in almost all of the lung lobes.

The changes in the volume of the blood vessels correlate well with regional ventilation, also derived from the functional CT based on lobe expansion. Vasodilation occurs 


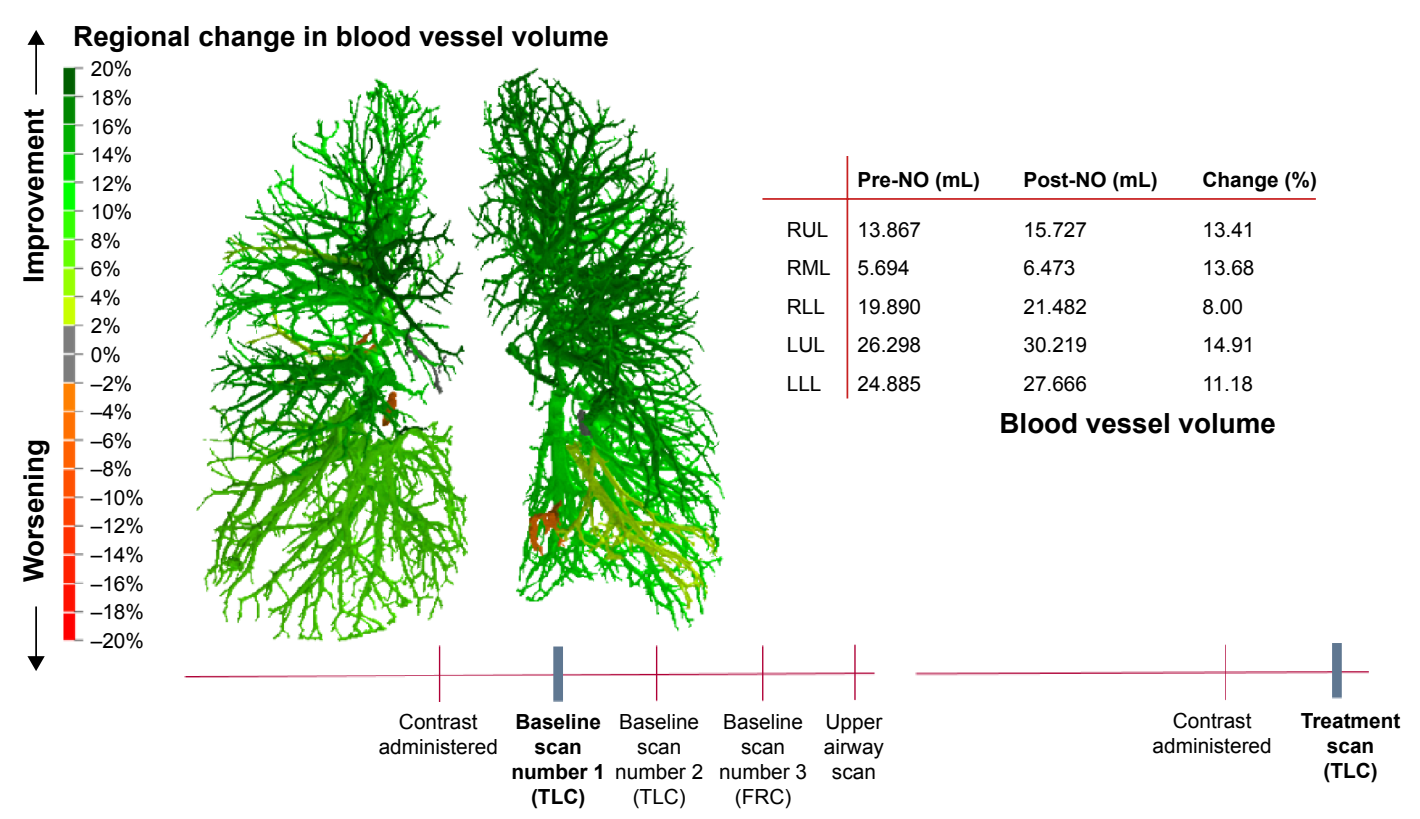

Figure 3 Segmented blood vessel model can be colored to display the regional change in volume after 20 minutes of iNO treatment.

Abbreviations: FRC, functional residual capacity; LLL, left lower lobe; LUL, left upper lobe; RLL, right lower lobe; RML, right middle lobe; RUL, right upper lobe; TLC, total lung capacity; pre-NO, pre-nitric oxide inhalation; post-NO, post-nitric oxide inhalation.

in areas that are well ventilated. Because of this, we can anticipate that the ventilation perfusion ratio is preserved (Figure 6). There was no decrease in the oxygen saturation (Figure 7), which confirms the fact that the vasodilation is in line with the ventilation. Therefore, this presumably reflects a preserved ventilation perfusion ratio. The patients improved in their subjective feelings of dyspnea and exercise tolerance 24 hours after treatment (Figure 8).

\section{Discussion}

Combined inhalation of $\mathrm{NO}$ and oxygen with a pulsed device for 20 minutes in patients with chronic PH due to COPD caused a significant vasodilation in a majority of the vessels visualized by FRI. The changes, mostly increases, in blood vessel caliber seem to occur in well-ventilated areas of the lung. There was no decrease in the oxygen saturation, suggesting that ventilation/perfusion ratio was preserved.

There is currently only one study, performed by Vonbank, ${ }^{14}$ which has used iNO. This study was a randomized controlled trial of 40 COPD patients with $\mathrm{PH}$. They were treated for 3 months with pulsed iNO combined with oxygen. Compared with oxygen alone, the combined inhalation caused a significant decrease in MPAP and pulmonary arterial resistance index, without decreasing arterial oxygenation. There was an increase in cardiac output, with preserved systemic hemodynamics. This was the first controlled randomized trial showing that inhalation of NO with oxygen can safely be used for treating $\mathrm{PH}$ in patients with COPD. The study was not focused on the underlying pathophysiological mechanisms. Vonbank et $\mathrm{al}^{14}$ nevertheless demonstrate that it is possible to give a small concentration of $\mathrm{NO}$ at the beginning of the inspiration. As a consequence, the exposure of the lung to NO is reduced to a very small volume and systemic overall toxicity of NO is avoided.

Delivery of NO in pulsed doses early in inspiration, using a short pulse width, has the potential to follow the flow and to be delivered to the well-ventilated zones. In our study, we could confirm that this is indeed what happens with this application mode.

NO-mediated vasodilation occurs via the activation of soluble guanylate cyclase, the production of cyclic guanosine monophosphate (cGMP), and subsequent relaxation of vascular smooth muscle. iNO produces pulmonary vasodilation with minimal effect on systemic vascular beds due to its high affinity for hemoglobin and rapid inactivation. Patients with end-stage COPD have impaired NO-mediated endothelium-dependent pulmonary artery relaxation ${ }^{9-12}$ because of a reduced expression of endothelial NO synthase. ${ }^{23,24}$ Several groups ${ }^{14,25,26}$ could show that short-term use of NO can improve the hemodynamics in COPD patients. The effect on the partial pressure of arterial oxygen $\left(\mathrm{PaO}_{2}\right)$ is however controversial. In some studies, $\mathrm{NO}$ lowers $\mathrm{PaO}_{2},{ }^{27}$ whereas in others, it improves the same, ${ }^{26}$ and in some other studies, there seems to be no effect. ${ }^{14}$ Therefore, there are probably several phenotypes for NO response in COPD. It is possible that the controversial 


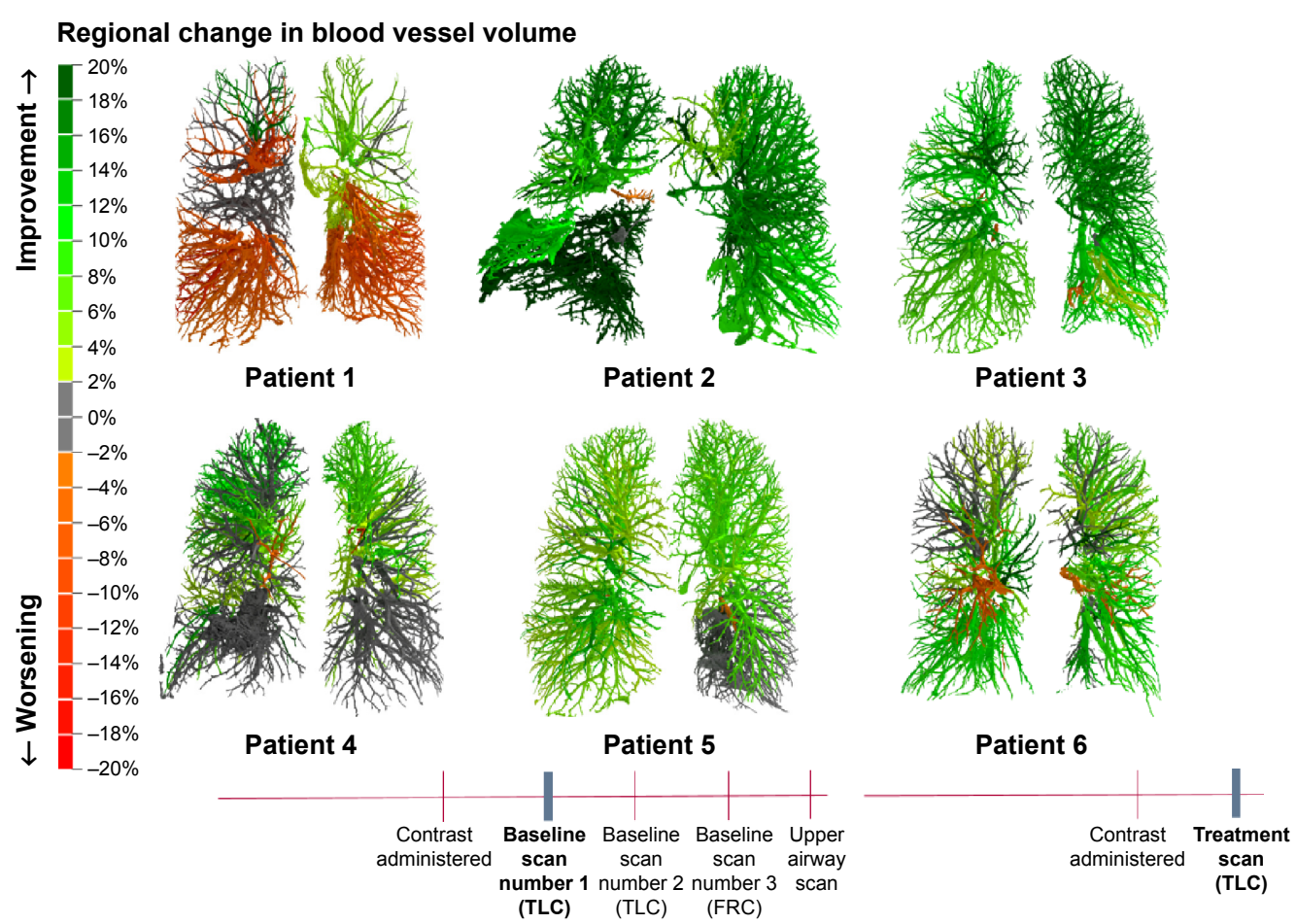

Figure 4 Regional changes in blood vessel volume.

Abbreviations: TLC, total lung capacity; FRC, functional residual capacity.

results are linked to the dosage of iNO used. Finding the right dose to reduce the mPAP and pulmonary vascular resistance (PVR) while maintaining $\mathrm{PaO}_{2}$ is a challenge..$^{14,27-30} \mathrm{In}$ a number of animal species and under several vasoconstrictive stimuli, ${ }^{31,32}$ iNO produced rapid and effective pulmonary vasodilation at concentrations between $5 \mathrm{ppm}$ and $80 \mathrm{ppm}$. Evaluations in the new-born lamb demonstrated that iNO selectively reverses hypoxic pulmonary vasoconstriction, with maximal pulmonary vasodilation produced by NO concentrations of $80 \mathrm{ppm}$. In an animal model of persistent $\mathrm{PH}$
A

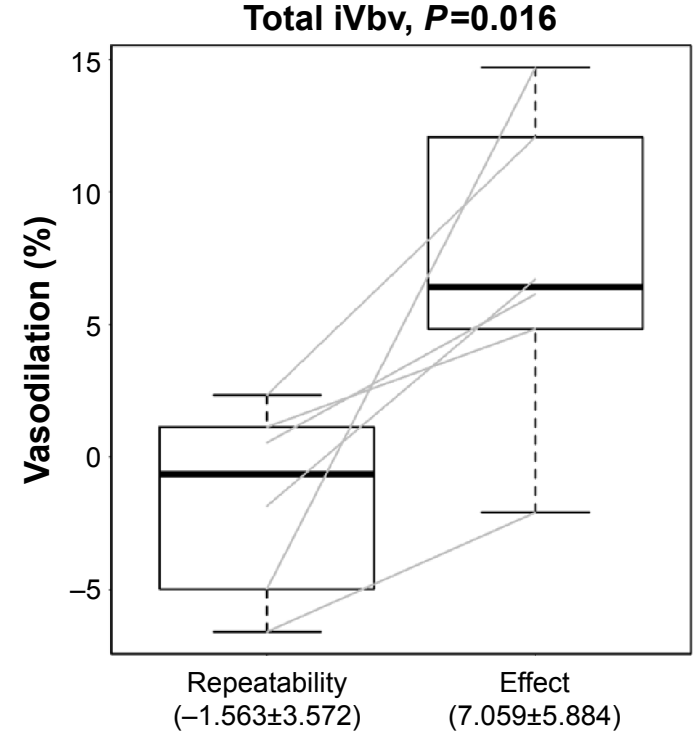

B

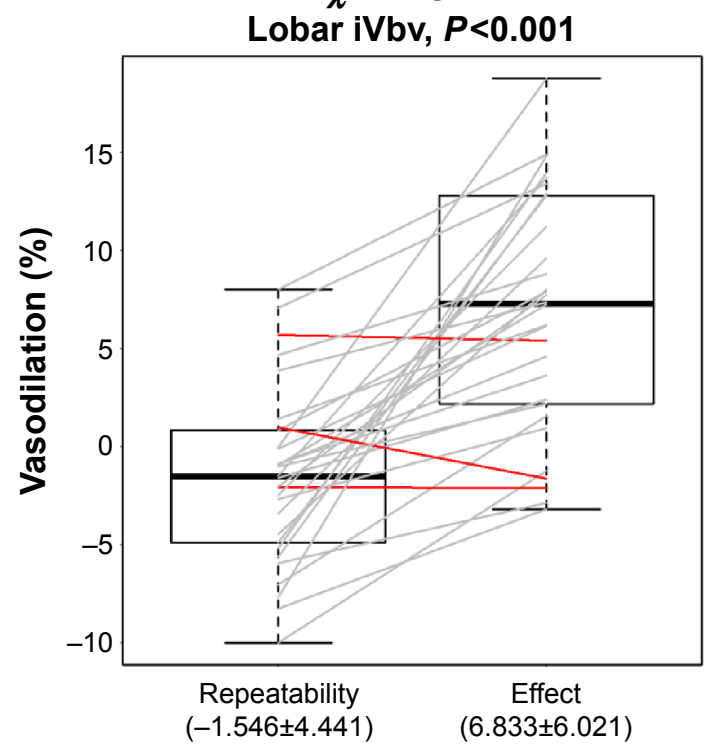

Figure 5 Repeatability of two TLC scans and total lung vasodilation (\%) and lobar vasodilation (\%).

Notes: (A) Vasodilation for the total lung: repeatability of the two TLC scans is $1.5 \%$, there is a total increase of $7 \%$ in the blood vessel volume using paired $t$-test ( $P=0.016)$. (B) Lobar vasodilation: repeatability of the two TLC scans is $1.5 \%$, there is a total increase of $6.8 \%$ of the blood vessel volume using paired $t$-test $(P<0.001)$. Gray lines: improvement in lobar blood volume; red lines: lobar blood volume remains stable or decreases.

Abbreviations: lobar iVbv, image-based blood vessel volume of the lobes; TLC, total lung capacity; total iVbv, image-based blood vessel volume of the total lung. 


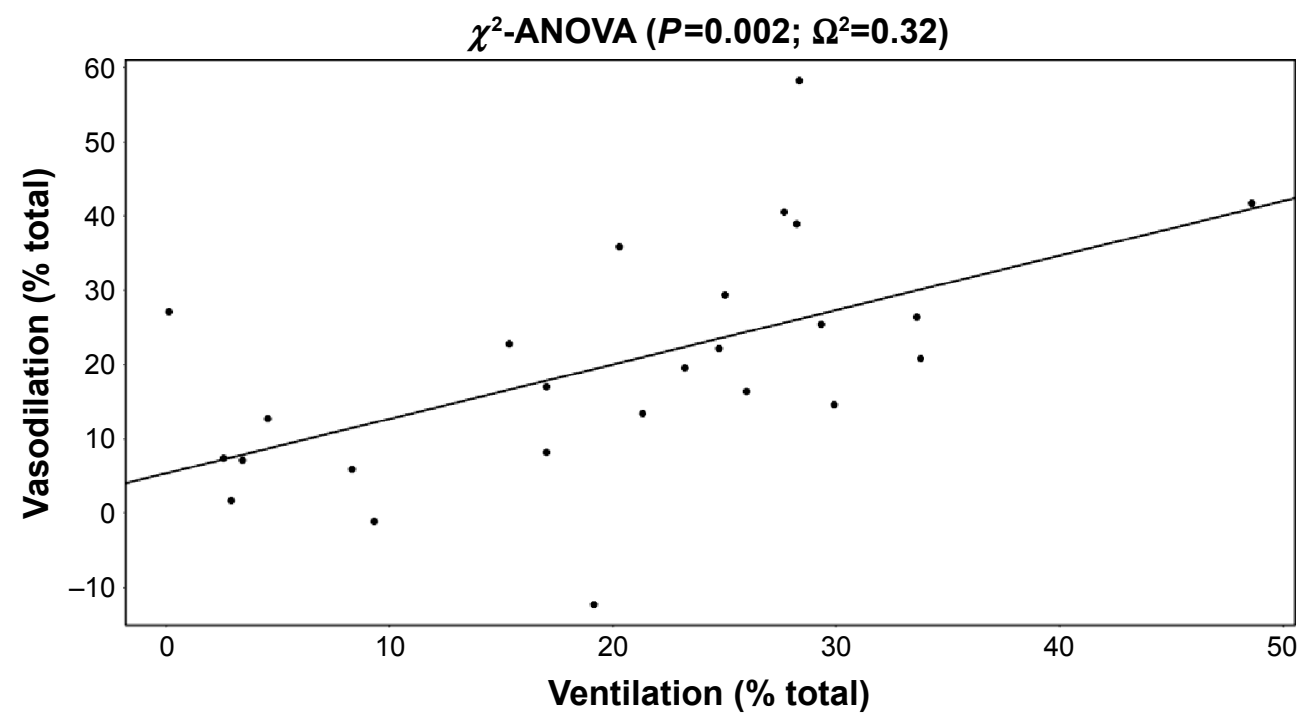

Figure 6 Correlation between ventilation and vasodilation.

Notes: $\Omega^{2}$ is similar to $R^{2}$ but for mixed models. Mixed models correct for the interdependence of lobes (five lobes in one patient).

of the newborn, neonatal lambs displayed a marked and rapid pulmonary vasodilation that improved oxygenation, at iNO concentrations of $100 \mathrm{ppm}$.

Too high doses of $\mathrm{NO},{ }^{28}$ however, may decrease the $\mathrm{PaO}_{2}$ due to worsening of the ventilation perfusion distributions, caused by a greater increase in blood flow compared to the increase in airflow reaching the vasodilated areas. Taking into account these earlier studies, our patients received $30 \mu \mathrm{g} / \mathrm{kg}$ IBW/hour doses, which obviously prevented excess vasodilation and lowering of ventilation/perfusion ratio (V/Q) ratio. This dose was chosen because it was the highest dose safely tested so far and the objective of this study was to observe with FRI the vascular effect induced by the strongest safe dose. Further studies are needed to determine the doseresponse relationship.

One can also hypothesize that the severity of the PH may play an essential role in the response to NO treatment. Our patients had relatively high severity of $\mathrm{PH}$, the mean SPAP was $49 \mathrm{mmHg}$. This was also confirmed by Katayama. ${ }^{29}$ They looked at the effect of NO in nine patients with chronic

\begin{tabular}{c|cc} 
Patients & Pre-iNO treatment & Post-iNO treatment \\
\hline Patient 1 & 96 & 97 \\
Patient 2 & 98 & 98 \\
Patient 3 & 99 & 99 \\
Patient 4 & 96 & 96 \\
Patient 5 & 94 & 94 \\
Patient 6 & 99 & 100 \\
& & $\mathrm{SpO}_{2}(\%)$
\end{tabular}

Figure 7 The $\mathrm{SpO}_{2}$ did not decrease after iNO. Abbreviations: iNO, inhaled nitric oxide; $\mathrm{SpO}_{2}$, oxygen partial pressure. obstructive disease, eleven patients with severe $\mathrm{PH}$, and 14 healthy volunteers. The patients were randomized for inhalation of 40 ppm NO or air for 20 minutes. There was a decrease in transcutaneous arterial oxygen tension in normal subjects and in patients with COPD overall. There was, however, no change in arterial oxygen tension in patients with severe $\mathrm{PH}$. The responding phenotype of COPD patients needs to have a relatively high PAP.

In our study, vasodilation occurred in most of the lobes after 20 minutes of pulsed iNO, which probably contributes to the lowering of the pressure because it represents an important vascular recruitment. It occurs in well-ventilated areas because it follows, as mentioned, the ventilation, preventing $\mathrm{V} / \mathrm{Q}$ mismatch, and oxygen desaturation. Finally, and probably, the most important observation was that the patients did feel better with less dyspnea and better exercise tolerance in the hours following the exposure to iNO. Our hypothesis is that heterogeneous, ventilationdriven vasodilation by pulsed iNO may lead to a reduction of cardiac afterload (= larger cardiac output) and better
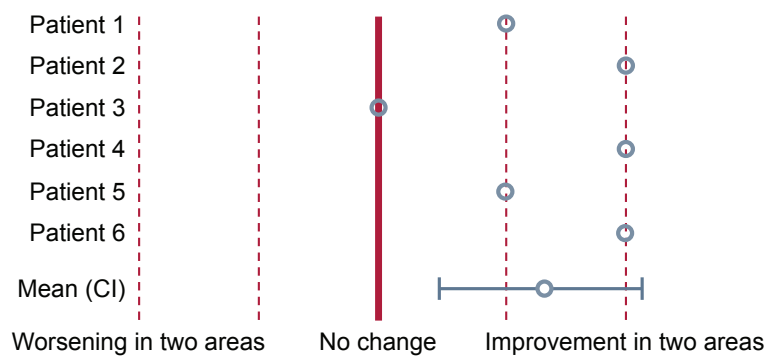

Figure 8 Patients' subjective feelings of dyspnea and exercise tolerance. Abbreviation: $\mathrm{Cl}$, confidence interval. 
oxygenation (preserved V/Q ratio), resulting in improvement of exercise tolerance in patients with combined COPD/PH. This hypothesis, however, needs to be confirmed in clinical confirmation studies.

We expect that pulsed NO could also be a promising treatment in other lung diseases leading to $\mathrm{PH}$, such as pulmonary fibrosis. This was confirmed by Channick et al. ${ }^{33}$ They showed that in a patient with pulmonary fibrosis and $\mathrm{PH}$, NO inhalation induced a significant improvement of arterial oxygenation and reduction in pulmonary vascular resistance. Therefore, in chronic lung disease in combination with severe $\mathrm{PH}$, iNO could have a potential therapeutic role as a selective pulmonary vasodilator.

\section{Conclusion}

In conclusion, using FRI, we were able to show a significant vasodilation of most of the vessels after iNO for a limited time period. In our patients, there was an average increase of blood volume of $7.06 \%$ after inhalation of pulsed NO, without a decrease in oxygen saturation, and patients tended to feel better after the treatment. Systemic treatments of $\mathrm{PH}$ showed no significant elevated $\mathrm{SpO}_{2}$ and improved healthrelated quality of life. The usefulness of these systemic vasodilator agents is limited due to their side effects and poor tolerance. ${ }^{34-36}$ Short-term treatment with pulsed NO in combination with oxygen could be a promising treatment for patients with COPD and PH. Further studies are needed to determine the effect of long-term continuous treatment with pulsed NO and oxygen in patients with COPD and $\mathrm{PH}$, especially considering exercise tolerance and quality of life. There is also need to investigate the effect of pulsed NO in other chronic lung diseases complicated by $\mathrm{PH}$, such as pulmonary fibrosis.

\section{Acknowledgment}

This study was supported by Bellerophon Therapeutics.

\section{Disclosure}

B Hajian is a pulmonologist at the University Hospital Antwerp. She is doing clinical research in the field of enhanced COPD phenotyping to improve treatment strategies. J De Backer, C Van Holsbeke, and W Vos and F Ferreira are affiliated with Fluidda NV. DA Quinn is affiliated to Bellerophon Therapeutics. The authors have no other relevant affiliations or financial involvement with any organization or entity with a financial conflict with the subject matter or materials discussed in the manuscript apart from those disclosed.

\section{References}

1. Minai OA, Chaouat A, Adnot S. Pulmonary hypertension in COPD: epidemiology, significance, and management: pulmonary vascular disease: the global perspective. Chest. 2010;137(6 suppl):39S-51S.

2. McLaughin VV, Hoeper MM. Pulmonary arterial hypertension: the race for the most effective treatment. Am J Respir Crit Care Med. 2005; 171(11):1199-1201.

3. Peinado VI, Barbera JA, Ramirez J, et al. Endothelial dysfunction in pulmonary arteries of patients with mild COPD. Am J Physiol. 1998; 274(6 pt 1):L908-L913

4. Peinado VI, Pizarro S, Barbera JA. Pulmonary vascular involvement in COPD. Chest. 2008;134(4):808-814.

5. Weitzenblum E, Mammosser M, Ehrhart M. Evolution and prognosis of pulmonary hypertension in chronic obstructive pulmonary diseases. Herz. 1986;11(3):147-154.

6. Weitzenblum E, Chaouat A, Kessler R. Pulmonary hypertension in chronic obstructive pulmonary disease. Pneumonol Alergol Pol. 2013; 81(4):390-398.

7. Melot C, Hallemans R, Naeije R, Mols P, Lejeune P. Deleterious effect of nifedipine on pulmonary gas exchange in chronic obstructive pulmonary disease. Am Rev Respir Dis. 1984;130(4):612-616.

8. Long term domiciliary oxygen therapy in chronic hypoxic cor pulmonale complicating chronic bronchitis and emphysema. Report of the Medical Research Council Working Party. Lancet. 1981;1(8222):681-686.

9. Dinh-Xuan AT, Higenbottam TW, Clelland CA, et al. Impairment of endothelium-dependent pulmonary-artery relaxation in chronic obstructive lung disease. $N$ Engl J Med. 1991;324(22):1539-1547.

10. Palmer RM, Ferrige AG, Moncada S. Nitric oxide release accounts for the biological activity of endothelium-derived relaxing factor. Nature. 1987;327(6122):524-526

11. Frostell C, Fratacci MD, Wain JC, Jones R, Zapol WM. Inhaled nitric oxide. A selective pulmonary vasodilator reversing hypoxic pulmonary vasoconstriction. Circulation. 1991;83(6):2038-2047.

12. Pepke-Zaba J, Higenbottam TW, Dinh-Xuan AT, Stone D, Wallwork J. Inhaled nitric oxide as a cause of selective pulmonary vasodilatation in pulmonary hypertension. Lancet. 1991;338(8776):1173-1174.

13. Pepke-Zaba J, Morrell NW. Pulmonary hypertension in patients with COPD: NO treatment? Thorax. 2003;58(4):283-284.

14. Vonbank K, Ziesche R, Higenbottam TW, et al. Controlled prospective randomised trial on the effects on pulmonary haemodynamics of the ambulatory long term use of nitric oxide and oxygen in patients with severe COPD. Thorax. 2003;58(4):289-293.

15. Abman SH, Chatfield BA, Hall SL, McMurtry IF. Role of endotheliumderived relaxing factor during transition of pulmonary circulation at birth. Am J Physiol. 1990;259(6 pt 2):H1921-H1927.

16. Shaul PW, Yuhanna IS, German Z, Chen Z, Steinhorn RH, Morin FC 3rd. Pulmonary endothelial NO synthase gene expression is decreased in fetal lambs with pulmonary hypertension. Am J Physiol. 1997; 272(5 pt 1):L1005-L1012.

17. Kinsella JP, Abman SH. Inhaled nitric oxide in the premature infant: animal models and clinical experience. Semin Perinatol. 1997;21(5): $418-425$.

18. Gross I. Recent advances in respiratory care of the term neonate. Ann N Y Acad Sci. 2000;900:151-158.

19. De Backer JW, Vos WG, Vinchurkar SC, et al. Validation of computational fluid dynamics in CT-based airway models with SPECT/CT. Radiology. 2010;257(3):854-862.

20. De Backer W, Vos W, Van Holsbeke C, et al. The effect of roflumilast in addition to LABA/LAMA/ICS treatment in COPD patients. Eur Respir J. 2014;44(2):527-529.

21. De Backer J, Vos W, Vinchurkar S, et al. The effects of extrafine beclometasone/formoterol (BDP/F) on lung function, dyspnea, hyperinflation, and airway geometry in COPD patients: novel insight using functional respiratory imaging. J Aerosol Med Pulm Drug Deliv. 2015; 28(2):88-99.

22. Xu R. Measuring explained variation in linear mixed effects models. Stat Med. 2003;22(22):3527-3541. 
23. Giaid A, Saleh D. Reduced expression of endothelial nitric oxide synthase in the lungs of patients with pulmonary hypertension. $N$ Engl $J$ Med. 1995;333(4):214-221.

24. Yang Q, Shigemura N, Underwood MJ, et al. NO and EDHF pathways in pulmonary arteries and veins are impaired in COPD patients. Vascul Pharmacol. 2012;57(2-4):113-118.

25. Germann P, Ziesche R, Leitner C, et al. Addition of nitric oxide to oxygen improves cardiopulmonary function in patients with severe COPD. Chest. 1998;114(1):29-35.

26. Yoshida M, Taguchi O, Gabazza EC, et al. Combined inhalation of nitric oxide and oxygen in chronic obstructive pulmonary disease. Am J Respir Crit Care Med. 1997;155(2):526-529.

27. Roger N, Barberà JA, Roca J, Rovira I, Gómez FP, Rodriguez-Roisin R. Nitric oxide inhalation during exercise in chronic obstructive pulmonary disease. Am J Respir Crit Care Med. 1997;156(3 pt 1):800-806.

28. Barberà JA. Nitric oxide in chronic obstructive pulmonary disease. Monaldi Arch Chest Dis. 1996;51(6):528-532.

29. Katayama Y, Higenbottam TW, Diaz de Atauri MJ, et al. Inhaled nitric oxide and arterial oxygen tension in patients with chronic obstructive pulmonary disease and severe pulmonary hypertension. Thorax. 1997; 52(2):120-124.

30. Moinard J, Manier G, Pillet O, Castaing Y. Effect of inhaled nitric oxide on hemodynamics and VA/Q inequalities in patients with chronic obstructive pulmonary disease. Am J Respir Crit Care Med. 1994;149(6): $1482-1487$.
31. Zayek M, Cleveland D, Morin FC. Treatment of persistent pulmonary hypertension in the newborn lamb by inhaled nitric oxide. $J$ Pediatr. $1993 ; 122(5$ pt 1):743-750.

32. Bland RD, Albertine KH, Carlton DP, MacRitchie AJ. Inhaled nitric oxide effects on lung structure and function in chronically ventilated preterm lambs. Am J Respir Crit Care Med. 2005;172(7):899-906.

33. Channick RN, Hoch RC, Newhart JW, Johnson FW, Smith CM. Improvement in pulmonary hypertension and hypoxemia during nitric oxide inhalation in a patient with end-stage pulmonary fibrosis. $\mathrm{Am} \mathrm{J}$ Respir Crit Care Med. 1994;149(3 pt 1):811-814.

34. Whyte KF, Flenley DC. Can pulmonary vasodilators improve survival in cor pulmonale due to hypoxic chronic bronchitis and emphysema? Thorax. 1988;43(1):1-8.

35. Mookherjee S, Ashutosh K, Smulyan H, Vardan S, Warner R. Arterial oxygenation and pulmonary function with Saralasin in chronic lung disease. Chest. 1983;83(6):842-847.

36. Blanco I, Santos S, Gea J, et al. Sildenafil to improve respiratory rehabilitation outcomes in COPD: a controlled trial. Eur Respir J. 2013; 42(4):982-992.
International Journal of COPD

\section{Publish your work in this journal}

The International Journal of COPD is an international, peer-reviewed journal of therapeutics and pharmacology focusing on concise rapid reporting of clinical studies and reviews in COPD. Special focus is given to the pathophysiological processes underlying the disease, intervention programs, patient focused education, and self management protocols.

\section{Dovepress}

This journal is indexed on PubMed Central, MedLine and CAS. The manuscript management system is completely online and includes a very quick and fair peer-review system, which is all easy to use. Visit http://www.dovepress.com/testimonials.php to read real quotes from published authors. 